Relations industrielles

Industrial Relations

\title{
Arbitrage en matière d'ancienneté
}

\section{Émile Gosselin}

Volume 13, numéro 3, juillet 1958

URI : https://id.erudit.org/iderudit/1022428ar

DOI : https://doi.org/10.7202/1022428ar

Aller au sommaire du numéro

\section{Éditeur(s)}

Département des relations industrielles de l’Université Laval

ISSN

0034-379X (imprimé)

1703-8138 (numérique)

Découvrir la revue

Citer ce document

Gosselin, É. (1958). Arbitrage en matière d'ancienneté. Relations industrielles / Industrial Relations, 13(3), 337-340. https://doi.org/10.7202/1022428ar

Tous droits réservés (C) Département des relations industrielles de l’Université Laval, 1958
Ce document est protégé par la loi sur le droit d'auteur. L’utilisation des services d'Érudit (y compris la reproduction) est assujettie à sa politique d'utilisation que vous pouvez consulter en ligne.

https://apropos.erudit.org/fr/usagers/politique-dutilisation/ 


\title{
JURISPRUDENCE DU TRAVAIL
}

\author{
A R B I T R A G E
}

\section{UN CAS D'ANCIENNETÉ}

\section{Emile Gosselin (avec la collaboration de Me Marc Lapointe, co-signataire, et la dissidence de Me Marcel Crête)}

\begin{abstract}
Il s'agit d'un grief concernant la mise en application de la clause d'ANCIENNETÉ inscrite dans la convention intervenue entre Shawinigan Chemicals Limited et le Syndicat national des Travailleurs en Produits chimiques de Shawinigan Falls, Inc.
\end{abstract}

Dans son exposé écrit devant le tribunal, le Syndicat allègue qu'à deux reprises la Compagnie a violé le paragraphe 804 de la convention collective existant entre les parties, ayant rempli trois postes de gardien sans tenir compte de la clause d'ancienneté.

Il s'agit en réalité de deux griefs enregistrés à des dates successives; mais vu que les deux cas semblaient identiques, les deux parties ont décidé d'un commun accord de les joindre en un seul et même grief.

La première partie du présent grief fait suite à la nomination par la Compagnie de Georges Degagné comme gardien à la division du Carbure, le 10 septembre 1956. Ce dernier était à l'emploi de la Compagnie depuis le 24 mai 1957. Le lendemain, quatre employés formulaient un grief auprès de la Compagnie pour violation de la clause d'ancienneté: 1) Elzéar Pelletier, à l'emploi de la Compagnie depuis le 25 avril 1912; 2) Eddy Diamond, employé depuis le 15 mai 1920; 3) Antonio Blais, dont l'ancienneté remonte au 17 novembre 1924; et 4) Alfred Martin, embauché par la Compagnie le 9 juillet 1926.

Dans le deuxième cas, Aurèle Biron, à l'emploi de la partie patronale depuis le 3 juin 1940, déposait un grief à l'encontre de la nomination de deux gardiens à la division de la Fonderie. Le premier, Jacques Aubry, travaillait pour la Compagnie depuis le 27 juillet 1940, et le deuxième, Léo Baudoin, depuis le 15 janvier 1942. Biron alléguait violation de la clause d'ancienneté.

Dans son propre mémoire, la partie patronale soutient qu'elle n'a pas violé la clause d'ancienneté de la convention collective.

804 - Dans tous les cas de promotion ou de déplacement, l'on accordera préférence à l'ancienneté de service avec la Compagnie dans le cas d'employés qualifiés, mais ces promotions ou ces déplacements seront effectués sur une base de division seulement.

Selon la Compagnie, «The Syndicate's claim is not subtantiated and... the two (2) aggrieved employees, namely, Pelletier and Biron, did not meet the qualification requirements for the position of Plant Guard ». Selon elle, les deux employés ne rencontraient pas les exigences physiques de la tâche. En outre, Biron ne possédait pas une scolarité suffisante. La Compagnie allègue qu'elle est en droit de déterminer les exigences personnelles que doivent désormais rencontrer les candidats au poste de gardien: 
Article II - Fonction de la Gérance

200 - Le Syndicat accepte que la gérance a le droit exclusif de prendre toute décision relative aux fonctions de la gérance et à la direction générale de l'usine, notamment:

a) embaucher les employés et les mettre à pied;

b) opérer l'usine, diriger le travail et attribuer des tâches aux employés;

c) choisir les produits à être fabriqués de même que les matériaux et l'outillage nécessaires à la production;

d) changer les méthodes de fabrication et la quantité de production;

e) ajouter, retrancher des tâches et changer leur contenu;

f) établir et mettre en force des règlements concernant la discipline.

La Compagnie ajoute qu'elle a entrepris un programme planifié de réglementation sécuritaire qui doit se réadiser par étapes jusqu'à ce que l'objectif ultime prévu soit accompli. En vue de cet objectif, il est de première nécessité, selon la Compagnie, de choisir des gardiens d'usine qui rencontrent les exigences requises, et il n'appartiendrait pas au tribunal de juger de la politique établie par la Compagnie au sujet des exigences requises pour le poste de gardien. La partie patronale conclut donc comme suit: «Management, in concluding its arguments in connection with this case, submits for the Board's consideration the following:

a) The Labor Agreement expresses agreement that it is an exclusive right of Management to take decisions related to Management's functions. One of Management's functions has always been, and must continue to be, that of setting job qualification requirements.

b) Management did adhere to the Labor Agreement provision concerning seniority preference in the case of employees who met the qualifications required by Management for the job. The word «qualified 》 as used in the Labor Agreement in connection with seniority rights (ref. Article VIII, paragraph 804) is not defined. Management has met the requirements of this particular provision as far as qualified is concerned by exercising one of its functions, namely, the setting up of qualification requirements.

c) Nothing in the Labor Agreement prevents or limits the Management with respect to establishing any methods, practices, policies, etc., that are not specifically conditioned by provision in the Agreement. 》

Nous croyons que le litige repose sur les questions suivantes: en vertu de la convention collective liant les parties en présence, la Compagnie peut-elle changer le contenu des tâches? Peut-elle modifier les exigences requises? Et dans l'un ou l'autre cas, quelles sont ses obligations vis-à-vis la partie syndicale, étant donné les clauses d'ancienneté et de règlement des griefs? A-t-elle violé la clause d'ancienneté?

En vertu de la convention maintenant en vigueur, c'est le droit de la Compagnie de changer le contenu des tâches, donc de modifier les exigences personnelles requises par les nouvelles tâches établies. Cependant, et nous croyons que c'est là un principe généralement établi dans les entreprises, il doit y avoir un rapport intime entre l'objet de la tâche, son contenu, et les exigences requises. Il est en preuve que la Compagnie est en voie d'élaborer une nouvelle politique de surveilvance de ses usines, et c'est là son droit. C'est également son droit de mettre en force cette politique en changeant le contenu de la tâche, et, par voie de conséquence nécessaire, de modifier les exigences nequises. Cependant, ces transformations, dont nous n'avons pas ici à juger de l'opportunité, doivent rencontrer les exigences fondamentales de la convention collective:

1) Le contenu de la tâche doit être effectivement modifié. 
2) Pour ne pas réduire à néant la valeur efficace des clauses d'ancienneté et de règlement des griefs, ce nouveau contenu doit être clairement expliqué aux employés. De plus, si de nouvelles exigences personnelles deviennent strictement nécessaires, les exigences totales de la tâche, accompagnées de critères de sélection aussi précis que possible, doivent être clairement indiqués aux employés.

Dans le présent cas, il semble que la nouvelle politique de surveillance établie par la Compagnie soit restée à l'état de projet. Le contenu de la tâche de gardien est resté sensiblement ce qu'il était. En outre, à supposer que la Compagnie se réclame du droit de hausser les exigences en vue d'une tâche dont le contenu et les responsabilités seront modifiés à brève échéance, il n'apparaît nulle part qu'on ait indiqué aux candidats recrutés ce que serait en fait la future tâohe, quant à son contenu et quant à ses nouvelles exigences.

L'avis du 13 août 1956 et celui du 24 septembre valent-ils comme une indication certaine que le contenu des tâches de gardien était changé? Nullement. I] ne se rapporte qu'aux qualifications requises. Les employés étaient donc en droit de penser, faute d'indications précises au contraire, qu'il s'agissait du même emploi qu'auparavant et avec son contenu habituel, d'autant plus que la preuve syndicale non contredite valablement indique qu'il n'y eut tout au plus que des changements mineurs.

L'avis du 13 août et celui du 24 septembre 1956 comportaient-ils une description nette et précise des exigences de la tâche? En outre, la Compagnie pouvaitelle publier des avis ainsi libellés? Sur le premier point, nous croyons que l'avis était défectueux. Les qualifications physiques requises, apparemment du moins, sont laissées à l'approbation du médecin. Ici, à la face même de l'avis, rien de précis ne pouvait permettre un contre-examen médical. La Compagnie a en outre déposé comme exhibit C-3 un mémo administratif du docteur Stevenson indiquant quels seraient objectivement les critères médicaux de sélection. Or si les avis affichés s'en rapportaient aux critères du médecin tels qu'établis dans son mémo, rien n'indique que tel mémo ait été porté à la connaissance générale de ses employés avant le 18 septembre. En fait, si la Compagnie a cru l'affichage des vacances nécessaire, elle aurait dû également afficher au même moment les normes médicales requises afin de permettre au syndicat et aux employés impliqués dans un grief d'ancienneté ou autre de procéder à une contre-preuve médicale.

On prétendra peut-être que les qualifications morales et intellectuelles étaient bien établies sur l'avis d'affichage. Si l'avis d'affichage indiquait des conditions que l'on entendait suivre rigoureusement, de sonte que le manquement à une seule devait disqual'ifier le candidat automatiquement, il semble juste et normal de croire que la Compagnie aurait dû faire un effort spécial pour définir rigoureusement tous ses termes, en particulier en ce qui a trait aux conditions physiques et morales. Car en un tel cas l'ouvrier a droit de savoir exactement tout ce qu'on exigera de lui; l'avis comportant des faiblesses sur ce point, il nous semble nui. Si la Compagnie entendait procéder de façon moins rigouneuse et pondérer les différents facteurs de qualification lors de l'examen des candidats, de sorte qu'une faiblesse dans un domaine pouvait être compensé par de meilleunes qualifications dans l'autre (ce qui semble le cas à cause du vague même de certaines qualifications), il faudrait alors ne pas tenir rigueur à un employé faible sur un point seulement. Il faudrait également savoir quels sont les éléments de pondération.

Maintenant, la Compagnie prétend que son avis est valable comme description des exigences requises, qu'elle a le droit de procéder ainsi, et qu'elle n'a violé en rien les dispositions de la convention collective. Supposons pour l'instant, pour fins de démonstration, que l'avis d'affichage ait contenu une description exacte des qualifications requises, de sorte que nul employé ou officier du syndicat n'ait pu entretenir des doutes sur les intentions de la gérance. Que vaut pareil avis?

Si nous en oroyons le témoignage même des témoins de la Compagnie, le contenu de la tâche reste encore à définir. Du moins, s'il l'est dans l'esprit de la gé- 
rance, les employés remplissant aujourd'hui cette tâche ne semblent pas en avoir été affectés. Bien plus, la Compagnie maintient à leur emploi de gardien des personnes ne possédant aucune qualification spéciale, sauf de ne pouvoir faire autre chose.

En d'autres termes, dans le cas des gardiens, le mot «qualifié » n’aurait plus de rapport avec une tâche précise, dont le contenu est certain et connu de tous. Ce mot ne désignerait que ce que la Compagnie voudrait bien lui faire dire. On ne serait plus alors qualifié par rapport à une tâche qui existe mais par rapport à une tâche éventuelle ou encore par rapport à une définition arbitraire des qualifications. Comment alors un ouvrier pourrait-il faire un grief, démontrer qu'il est qualifié pour remplir une tâche, si celle-ci n'est qu'à l'état de projet? Comment alors pour un tribunal faire le rapprochement entre les qualités de l'individu et celles requises par la tâche, si cette dernière n'est pas encore bien établie?

Nous croyons qu'il est du domaine de la gérance d'établir les qualifications requises pour une tâche donnée, mais encore le lien entre le contenu et les qualifications doit être maintenu dans des limites raisonnables. Si la Compagnie prétendait, ce qui n'est nullement le cas, manipuler les conditions requises des individus sans égard au contenu des tâches, la procédure de règlement des griefs et la clause d'ancienneté ne tiendraient plus, n'auraient plus aucun sens. En d'autres termes, la Compagie nommerait les employés d'abord, selon des critères qu'elle seule connaîtrait, et distribuerait les fonctions ensuite. Ce qui est à l'encontre de tout usage.

En dernier lieu, l'avis d'affichage donne aux candidats, dans un cas un délai de 7 jours, dans l'autre, de 8 jours, pour signer leur formule de demande d'emploi. Il semble bien que ceux qui s'inscriraient après, ou qui de toute façon se sentiraient lésés dans leur ancienneté par une nomination, n'auraient pas de droit à la position, s'ils n'avaient au préalable rempli la formule de demande. Nous ne trouvons aucune disposition dans la convention permettant à la Compagnie de modifier indirectement les stades et les délais prévus clairement par la procédure des griefs. Si l'avis est valable, le grief naîtrait du dernier jour permis pour la signature de la formule, et seuls ceux qui l'auraient signée seraient admis à déposer un grief. Or il s'agit ici d'un grief d'ancienneté, et ce dernier ne peut naître que du jour où en fait une nomination est faite en violation de ladite clause. Nous ne nions pas le droit de la Compagnie d'annoncer des vacances et même de souligner les qualifications requises, si le contenu des tâches est bien connu, mais nous ne pouvons permettre que la clause d'ancienneté soit subordonnée à des délais unilatéralement établis par la Compagnie par voie d'affichage ou autrement. De sorte qu'un employé qui se croit lésé par une nomination peut, en suivant les délais prévus par la convention collective, se plaindre dès que l'acte est posé par la Compagnie, même s'il n'a pas suivi les délais prescrits par l'affichage des vacances. Les règlements de régie interne établis par la Compagnie pour une meilleure administration de son personnel ne peuvent en aucun cas mettre de côté des droits clairement et contractuellement établis par les parties.

La Compagnie a le droit de changer le contenu de la tâche de gardien et, par voie de conséquence, de modifier les exigences requises. Cependant, le contenu n'était pas sensiblement changé en date du 18 septembre, et de toute façon la Compagnie n'a pas annoncé de façon claire et précise qu'elle entendait établir une nouvelle tâche à brève échérance et quels en seraient le contenu et les exigences requises. En outre, l'avis d'affichage est nul en ce qu'il ne décrit pas suffisamment les nouvel'es exigences requises avec des critères permettant, au total, aux employés de vérifier leurs propres aptitudes et de contredire les décisions de la Compagnie. L'avis viole également les clauses relatives au règlement des griefs et à l'ancienneté.

Pour ces motifs, la Compagnie nommera Elzéar Pelletier au poste de gardien à la division du Carbure, et Aurèle Biron à la division de la Fonderie, avec pleine compensation pour salaires perdus à date, à compter des nominations faites par la Compagnie des individus qu'ils dépassent en ancienneté. 